\title{
Damage evolution in compacted graphite iron during thermomechanical fatigue testing
}

\author{
Viktor Norman, Peter Skoglund and Johan Moverare
}

\section{Linköping University Post Print}

\section{Tweet}

N.B.: When citing this work, cite the original article.

Original Publication:

Viktor Norman, Peter Skoglund and Johan Moverare, Damage evolution in compacted graphite iron during thermomechanical fatigue testing, 2015, International Journal of Cast Metals Research.

http://dx.doi.org/10.1179/1743133615Y.0000000019

Copyright: Maney Publishing

http://maneypublishing.com/

Postprint available at: Linköping University Electronic Press

http://urn.kb.se/resolve?urn=urn:nbn:se:liu:diva-121028 


\title{
Damage evolution in compacted graphite iron during thermomechanical fatigue testing
}

\author{
Viktor Norman $^{\mathrm{a} *}$, Peter Skoglund ${ }^{\mathrm{b}}$ and Johan Moverare \\ ${ }^{a}$ Division of Engineering Materials, Department of Management and Engineering, Linköping \\ University, SE-58183 Linköping, Sweden.

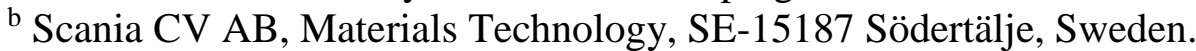 \\ ${ }^{c}$ Division of Engineering Materials, Department of Management and Engineering, Linköping \\ University, SE-58183 Linköping, Sweden. \\ *Corresponding author, phone 004613 284695, viktor.norman@liu.se, Linköping university, SE- \\ 58183 Linköping, Sweden
}

\begin{abstract}
Thermomechanical fatigue properties of a compacted graphite iron in an out of phase configuration are investigated for different maximum temperatures and mechanical strain ranges. Furthermore, the stress-strain hysteresis loops are analysed and in particular the unloading modulus, i.e. the elastic modulus measured during specimen unloading, is obtained from each cycle. This material parameter has earlier been explicitly related to the amount of microcracking in cast irons. The results show that the unloading modulus linearly declines with the numbers of cycles in all tests performed. In addition, the rate of change of the unloading modulus is closely related to the number of cycles to failure. Accordingly, it is concluded that microcracks are independently propagated by fatigue until a point of rapid crack-linking resulting in the ultimate failure. This is supported by microstructural analyses consisting of optical microscope images taken at different stages throughout the life of a specimen.
\end{abstract}

\section{Keywords}

Thermomechanical fatigue, Out-of-phase loading, Compacted graphite iron, Microcracking, Unloading elastic modulus.

\section{Introduction}

Cast iron is the most commonly used material in diesel engine components, such as the cylinder head in heavy-vehicle manufacturing. ${ }^{1,2}$ The motive of this material selection is normally argued to be the low material price and the associated simplicity of producing components with complicated geometry through casting.

As engine components are developed and designed information regarding the fatigue and failure resistance properties is often required in order to optimize the service lifetime. In general, this can be done in two ways, either through component testing or through component simulation using FEM tools; the latter requiring constitutive and lifetime assessment material models. This approach is regarded as the most efficient, leading to shorter product development times and lower resource consumption. Accordingly, the long term objective is to improve and extend the material modelling of cast irons for automotive applications.

Regarding the components at hand, material modelling is a challenging task due to the complex environment inside and close to the engine combustion chamber. Lately, special attention has been paid on the slow heating up and cooling down cycle corresponding to the start-stop cycle of the engine since this is believed to be highly detrimental and the dominating life-limiting mechanism ${ }^{2}$. This motivates the use of thermomechanical fatigue (TMF) testing which simulates the superposition of a thermal and a mechanical load. Such experimental data will constitute an input for the mentioned material modelling that is to be done.

The aim of the present paper is two-folded, (i) to report the thermomechanical fatigue properties of the compacted cast iron EN-GJV 400, and (ii) to propose an experimentally supported explanation on how damage evolves during cyclic loading. The latter is thought as a general 
formulation having the potential to also be descriptive for other kinds of cast irons, as well as being a starting point for the lifetime assessment modelling.

\subsection{Thermomechanical fatigue}

Thermomechanical fatigue (TMF) is commonly defined as the permanent and successive damage accumulated in a material due to an applied cyclic mechanical load and temperature. The induced thermal expansion complicates the stress-strain analysis which is overcome by the introduction of the mechanical strain, which is the component of strain consisting of the elastic and inelastic strain parts.

$$
\varepsilon_{T o t}(t)=\varepsilon_{E}(t)+\varepsilon_{I}(t)+\varepsilon_{T h}(t)=\varepsilon_{M e c h}(t)+\varepsilon_{T h}(t)
$$

The mechanical strain is solely responsible for the current stress in the material and should be regarded as the relative strain component with reference to the unstressed and thermally expanded state. Thus, the applied cyclic mechanical load is represented by the cyclic variation in the mechanical strain.

In this investigation, the cyclic mechanical strain and temperature variables differ with a 180 degrees phase shift, i.e. the maximum temperature coincides with the minimum mechanical strain, since this corresponds to the load situation normally felt in engine materials ${ }^{1}$. This is often referred to as out-of-phase (OP) TMF testing. An example of this is a rigidly clamped specimen, i.e. zero total strain, exposed to thermal cycling which results in an equal mechanical and thermal strain in magnitude but opposite in sign. It is useful to relate a TMF test with an arbitrary mechanical strain signal to this specific case by the reference to the ratio of the mechanical and thermal strain range,

$$
x=\frac{\Delta \varepsilon_{M e c h}}{\Delta \varepsilon_{T h}}
$$

which equals unity in the case of rigid clamping. This value can be thought of as the level of thermal expansion resistance, i.e. the portion of the thermal expansion which is impeded by the surrounding construction. Typically, the material inside an engine component should experience an $\mathrm{x}$-value lower than one since it is reasonable to assume that not all thermal expansion is prevented. However, geometrical features and residual stresses may still imply local x-values larger than unity.

There are not much published on TMF testing of compacted graphite iron except for a series of investigations done by Ghodrat et al. for notched specimens. ${ }^{3}$

\subsection{Deformation and damage in cast irons}

The deformation of cast irons has been extensively studied in the past by many investigators. It has been well recognized that the deformation behaviour is highly influenced by the interaction between the free graphite phase and the iron-rich matrix, ${ }^{4,5}$ which constitutes the typical microstructure of cast irons (see Figure 1a).

Even at very low levels of strain, debonding of the graphite phase from the matrix is often observed, regardless of the type of cast iron. ${ }^{4}$ In addition, internal cracking within the graphite occurring at the same order of strain has been reported. ${ }^{5}$ These two phenomena, graphite debonding and internal fracture, are considered as damage and will be referred to as microcracking throughout this paper. In this context, a microcrack is defined as a crack which only extends over a single interconnected graphite phase particle (i.e. a flake, nodule, etc.).

Haenny and Zambelli ${ }^{6}$ discussed microcracking in grey cast iron by relating it to concepts of the elastic modulus, namely the unloading modulus which is measured during partial unloading somewhere in a stress-strain curve. In accordance to even earlier investigations, ${ }^{7}$ they observed a consistent three-stage dependence of the unloading modulus on the stress level, regardless of the type of grey cast iron. In the first low-stress stage, the unloading modulus is indifferent while in the second and third it is decreasing linearly with increased stress. However still in need of further 
evidence, they argued that such behaviour is readily explained by the concept of successive microcracking of the graphite phase and the resulting local plasticity in the adjacent matrix.

Accordingly, the first assumption of this investigation is that microcracking is occurring homogenously over the gauge length during straining. These short cracks are believed to act as starting points for fatigue crack growth propagating through the matrix ligaments situated between adjacent graphite flakes. At some point, these microcracks will be linked up and a crack of a higher order will rapidly take form; a process which will be denoted as microcrack linking in the following. As a consequence, it is hypothesized that the amount of microcracking can be monitored by evaluating the unloading modulus in the load reversals throughout a TMF test.

Such a fracture process is indicated in the investigations made by Fash, ${ }^{8}$ and by Nisitani and Tanaka, ${ }^{9}$ who studied crack propagation on the specimen surface during fatigue tests using replica techniques. Independently, both groups concluded that (micro-)cracking is occurring throughout the whole life of the specimen and that the time required for crack nucleation is negligible. Furthermore, Pirgazi, Ghodrat and Kestens ${ }^{10}$ recently published an investigation of the crack path morphology of a thermomechanically fatigued CGI specimen. They observed that the fracture surface manifested a higher density of graphite particles than other arbitrarily selected crosssectional planes. Thus, it is reasonable to assume that the plane containing the most graphite particles will be the most sensitive to microcracking; thereby becoming the most likely final crack plane.

(a)

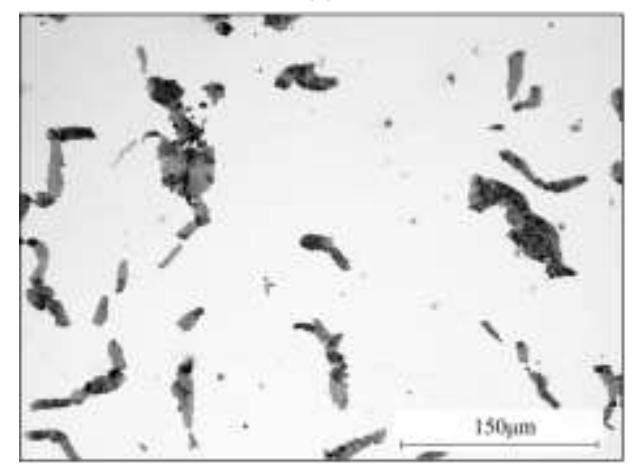

(b)

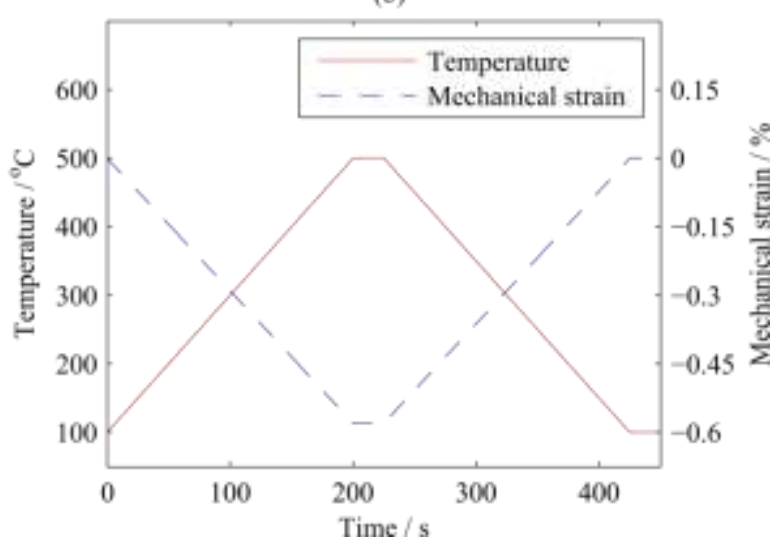

$1 a$ Optical microscope image of characteristic microstructure of EN-GJV 400 and $b$ shape of applied mechanical strain and temperature cycles

\section{Experimental Procedure}

\subsection{Material}

The investigated material is a pearlitic compacted graphite iron (CGI), EN-GJV 400, having the chemical composition displayed in Table 1. The specimens were cut from the inside of a cast block, i.e. from regions solidified under the same cooling conditions, and then machined into the desired specimen geometry. An image of the characteristic microstructure is presented in Figure 1a. Using the image process software Axiovision the corresponding nodularity was estimated as $10.05 \%$.

\subsection{Static tests}

Tensile tests were carried out at room and elevated temperatures, namely at $22^{\circ} \mathrm{C}, 100^{\circ} \mathrm{C}, 400^{\circ} \mathrm{C}$, $450^{\circ} \mathrm{C}$ and $500^{\circ} \mathrm{C}$, using an Instron 5982 electromechanic tensile test machine with a $100 \mathrm{kN}$ load cell. The strain rate applied was $0.02 \% \mathrm{~s}^{-1}$. The strain was measured using an external Instron $7361 \mathrm{C}$ extensometer and an Instron SF16 furnace was utilised to apply the desired temperatures. The specimens were of the same geometry as the specimens used in the thermomechanical testing, see below. 
The elastic modulus obtained from a static test was calculated as the slope of the stress-strain curve over a stress interval of $40 \mathrm{MPa}$ to $80 \mathrm{MPa}$. This value was also used in the calculation of the $0.02 \%$ offset yield strength.

\subsection{Thermomechanical fatigue tests}

The thermomechanical fatigue tests were conducted according to a validated code of practice ${ }^{11}$ in a strain-controlled configuration using an Instron 8801 servo hydraulic test frame. The temperature variation was generated through induction heating in combination with convection cooling by compressed air distributed onto the specimen through three nozzles.

The applied temperature and mechanical strain cycles consisted of a 200 seconds ramping up and down, as well as 25 seconds hold time at each turning point, see Figure 1b. Thus, the complete cycle was 450 seconds long. The tests were performed in an OP configuration, implying a 180 degree shift of the mechanical strain and temperature signal. Moreover, the mechanical strain value at the minimum temperature $\left(T_{\mathrm{Min}}\right)$ was always chosen as $0 \%$, i.e. the $R_{\varepsilon}$ was set to the negative infinity for all tests performed. Regarding the lower temperature value, it was chosen to a fixed value of $100^{\circ} \mathrm{C}$ except for a few tests. The reason for not selecting room temperature as this value was the experimental difficulty to cool the specimen at a specified rate below $100^{\circ} \mathrm{C}$.

The specimen geometry was cylindrical with a $6.3 \mathrm{~mm}$ diameter and a $25 \mathrm{~mm}$ parallel length, hence fulfilling the requirements specified in the code of practice ${ }^{11}$.

The failure criterion was also selected in accordance with the code-of-practice ${ }^{11}$ as the $10 \%$ drop in the stress range from a tangent draw at the last point of zero curvature in the stress range versus number of cycles plot.

\subsection{Hysteresis loop analysis}

The test variables including the stress and mechanical strain were recorded continuously and data was acquired twice every second in order to plot the hysteresis loops, i.e. the stress evolution in each cycle as a function of the mechanical strain. In addition, the unloading modulus was calculated in both the tensile and compressive side; including the first compressive load sequence even though it is not strictly an unloading modulus. The stress-strain slope was calculated over an interval of $0.04 \%$ starting from the first point of unloading.

\subsection{Microstructural investigations}

Qualitative microstructural investigations were performed using a Nikon Optiphot optical microscope, using 400 to 1000 times magnification. At first, the microstructure of an untested material was studied in order to determine the graphite structure and nodularity. Secondly, microcrack investigations were performed on fatigued specimens applied to the same TMF condition, namely $100-500^{\circ} \mathrm{C}$ and a mechanical strain ranging between $-0.37 \%$ and $0 \%$. One specimen was stopped after $50 \%$ of the total life (determined by a previous test) and a second specimen after $95 \%$. These cylindrical specimens were cut along the longitudinal axis, and then cut again in a cutting plane perpendicular to the first in order to have two different cross-sectional surfaces to study. All the cross-sectional surfaces studied were ground and polished stepwise following a standard program for cast iron materials.

Table 1 Measured chemical composition of EN-GJV 400/wt-\%

\begin{tabular}{llllllllll}
\hline $\mathbf{C}$ & $\mathbf{S i}$ & $\mathbf{M n}$ & $\mathbf{S}$ & $\mathbf{P}$ & $\mathbf{C r}$ & $\mathbf{N i}$ & $\mathbf{C u}$ & $\mathbf{V}$ & $\mathrm{As}$ \\
\hline 3.38 & 1.90 & 0.374 & 0.010 & 0.019 & 0.025 & $<0.050$ & 0.97 & 0.013 & 0.012 \\
\hline
\end{tabular}




\begin{tabular}{llll}
\hline $\boldsymbol{T} /{ }^{\circ} \mathbf{C}$ & $\boldsymbol{E} / \mathbf{G P a}$ & $\boldsymbol{R}_{\boldsymbol{p 0 . 0 0 2}} / \mathbf{M P a}$ & $\boldsymbol{R}_{\boldsymbol{m}} / \mathbf{M P a}$ \\
\hline 22 & 154 & 252 & 476 \\
100 & 148 & 248 & 474 \\
400 & 147 & 202 & 404 \\
450 & 139 & 199 & 370 \\
500 & 92 & 187 & 322 \\
\hline
\end{tabular}

(a)

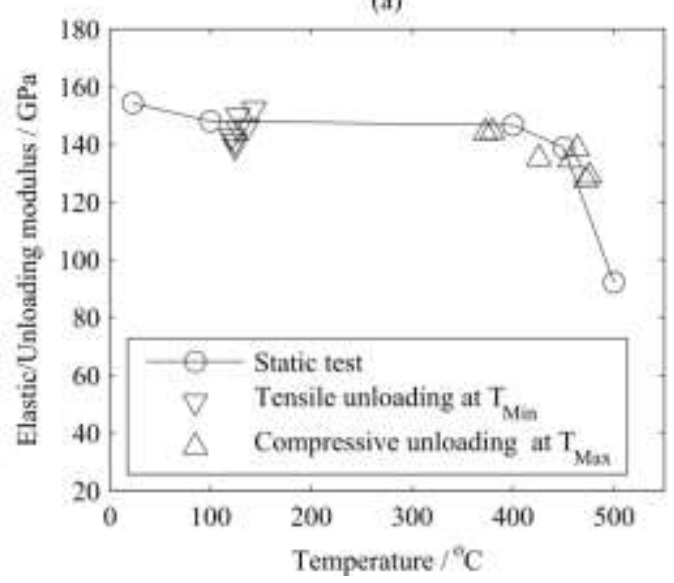

(b)

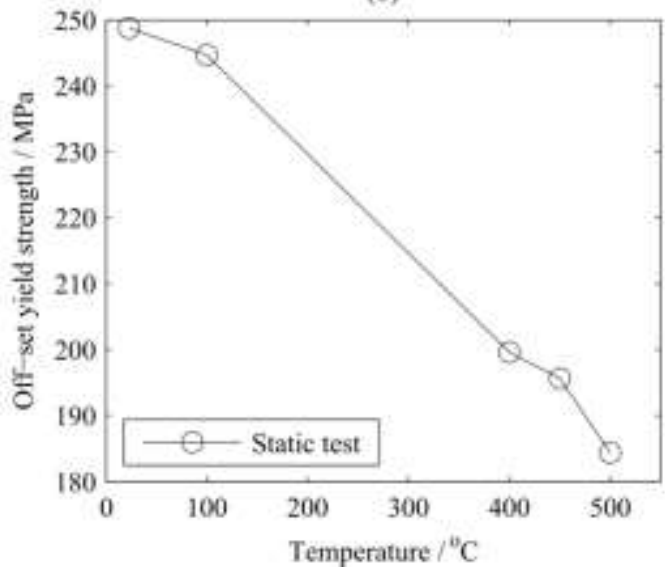

2 Temperature dependence of $a$ elastic and unloading modulus and $b$ off set yield strength

(a)

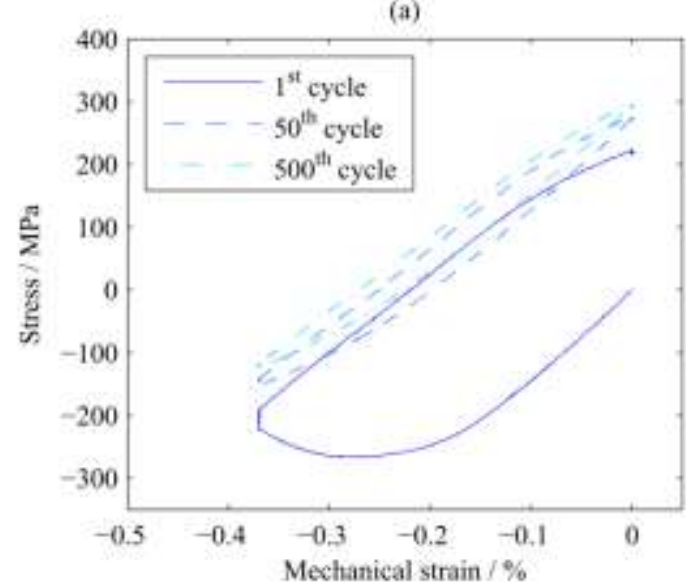

(b)

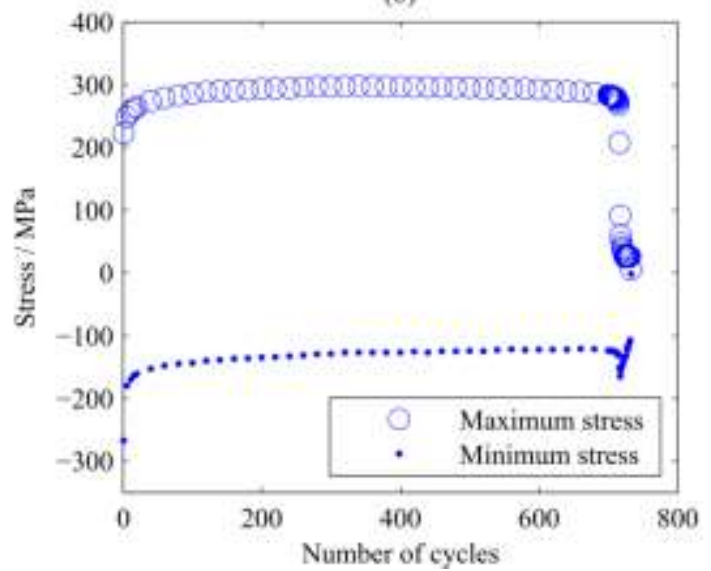

$3 a$ First, $50^{\text {th }}$ and $500^{\text {th }}$ hysteresis loop and $b$ maximum and minimum stress in each cycle of TMF test with $100-500^{\circ} \mathrm{C}$ and $0.37 \%$ mechanical strain range

\section{Results and Discussion}

\subsection{Static mechanical properties}

The results of the static tests are given in Table 2 and the temperature dependence of the elastic modulus and the off-set yield strength are illustrated in Figure 2. Both the elastic modulus and the off-set yield strength are temperature dependent within the tested temperature interval. The off-set yield strength decreases continuously with temperature while the elastic modulus is fairly indifferent for low temperatures but it drops significantly above $400^{\circ} \mathrm{C}$.

\subsection{Thermomechanical fatigue properties}

Characteristic TMF hysteresis loops are shown in Figure $3 \mathrm{a}$ corresponding to a test with a temperature ranging between $100^{\circ} \mathrm{C}$ to $500^{\circ} \mathrm{C}$ and a mechanical strain between $-0.37 \%$ and $0 \%$. Moreover, the maximum and minimum stress for each hysteresis cycle is plotted in Figure $3 \mathrm{~b}$. As in all other fatigue tests performed, the maximum and minimum stress increase similarly resulting in a 
fairly constant stress range, which in turn, varies with the applied mechanical strain range and temperature. Thus, the hysteresis loops clearly demonstrate a significant shake-up tendency but negligible strain hardening.

Strain range versus number of cycles to failure plots were produced for a couple of maximum temperature values, which are presented in Figure 4, where the solid markers represent a mechanical strain range corresponding to $\mathrm{x}$-value equal to unity. The effect of changing the mechanical strain and the maximum temperature is clearly demonstrated. In particular, the effect of increasing the temperature for a fixed $\mathrm{x}$-value (the solid dots) is more significant than solely increasing the equivalent level of mechanical strain associated with the temperature increase (e.g. the diamond dots).

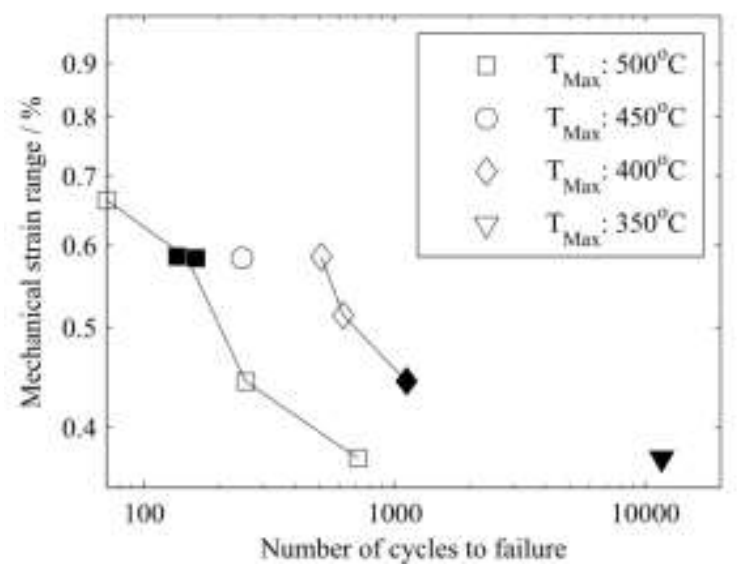

4 Strain life curves for different values of maximum temperature; solid markers represent mechanical strain range corresponding to $x$ value equal to unity

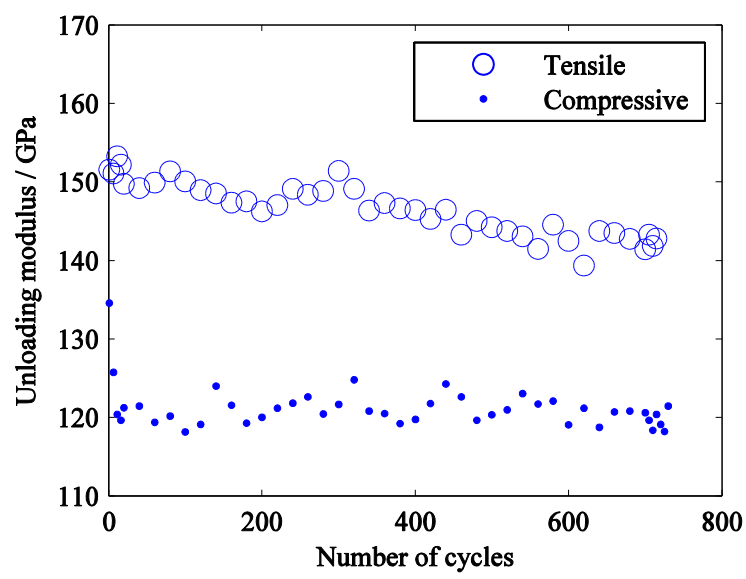

5 Unloading modulus in tension and compression as a function of number of cycles of TMF test with $100-500{ }^{\circ} \mathrm{C}$ and $0.37 \%$ mechanical strain range (same specimen as in Figure 3) 
(a)

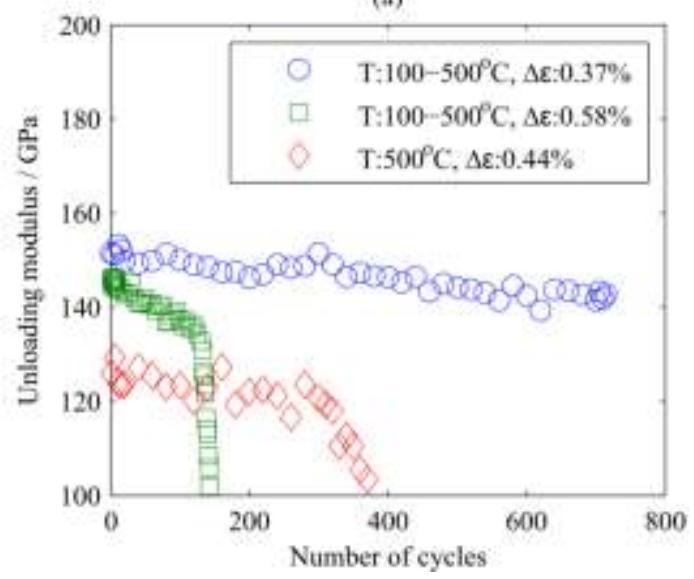

(b)

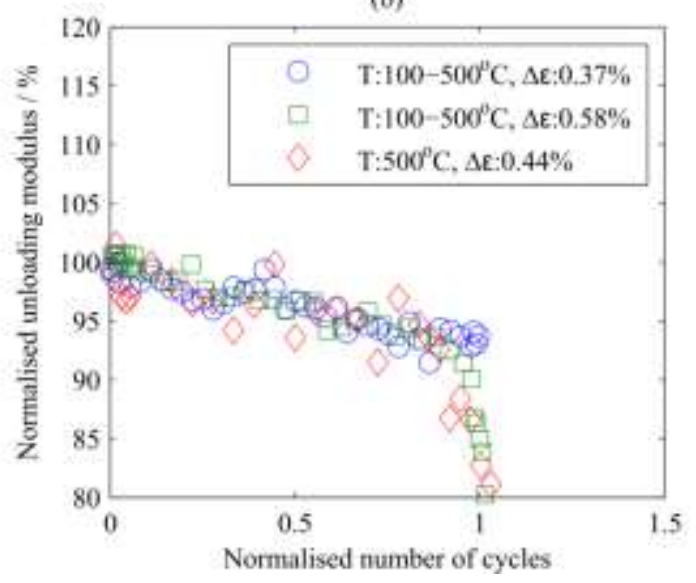

6 U Unloading modulus in tension of two TMF and one isothermal fatigue test as function of number of cycles $b$ Unloading modulus and number of cycles are normalized to unloading modulus of first cycle and number of cycles of failure $N_{f}$ respectively

\subsection{Unloading elastic modulus}

The unloading elastic modulus in tension and compression is plotted versus the number of cycles for a representative test specimen in Figure 5. This figure indicates that the unloading modulus in tension declines with a constant rate over the major part of the specimen life. The same behaviour is obtained for all TMF tests performed within this work, including isothermal low cycle fatigue tests at $500^{\circ} \mathrm{C}\left(T_{\min }=T_{\max }\right)$, as seen in Figure $6 \mathrm{a}$. This observation indicates that damage, i.e. loss in loadcarrying area, is induced in a constant rate until a point of instability is reached where both the stress and unloading modulus fall substantially. In addition, if the $\mathrm{x}$ - and y-axis are normalized with reference to the number of cycles to failure and the starting value of the unloading modulus respectively, the curves coincide as seen in Figure 6b. Thus, it is argued that all specimens, both TMF and low-cycle isothermal tests, follow the same physical process but at different rates depending on the magnitude of the load variables.

It was not possible to find an equally clear trend by a similar analysis of the compressive unloading modulus. However in most cases, the compressive unloading modulus was fairly constant such as in Figure 5. This seems reasonable since the crack surfaces should be partly closed during the compressive part of the cycle and thereby being capable of carrying a compressive load as opposite to a tensile load.

Some specimens did not fracture in two separate parts after the characteristic stress drop. These survivors are possibly explained by a partially through-cracked geometry consisting of some ligaments still intact, which due to the strain-controlled set-up only results in the opening and closing of large cracks when the specimen is completely fatigued.

The unloading modulus in tension and compression are compared to the elastic modulus obtained from the static test in Figure 2a. Apparently, the unloading modulus follows the same dependence on temperature as the elastic modulus measured in static tests.

The constant rate of change of the unloading modulus in percent per cycle is correlated to the number of cycles to failure for each specimen in Figure 7. This correlation follows a linear trend with a slope of minus one when plotted with logarithmic axes. The rate of change of the unloading modulus in each test was determined through a linear regression of the data between the first cycle and the number of cycles to failure determined by the failure criterion stated above.

The slope of minus one seen in Figure 7 is readily explained by the fact that the total percental loss in unloading modulus to fracture appears to be the same regardless of the applied test parameters (see Figure 6b), i.e.

$$
\Delta E=\frac{d E}{d N} N_{f}=\text { constant }
$$


which leads to the same expression as the trend seen in Figure 7.

The point found in the lower right corner was a specimen tested at low maximum temperature and mechanical strain range which resulted in 11670 cycles to failure. An estimate of this was calculated as 15596 by the evaluating the rate of change of the unloading modulus already after 2000 cycles. Thus, it is to some extent possible to estimate a long-lived specimen at an early stage by this relation.

\subsection{Microcrack investigation}

As the first observation, microcracks emanating from graphite flakes are found sporadically over the cross-sectional surface of a fatigued specimen, both after $50 \%$ and $95 \%$ of the total lifetime, as illustrated with a few examples in Figure 8. Thus, simultaneous microcrack growth is observed near the surface and in the bulk, as well as at different longitudinal positions on the specimen. The assumption of homogenous microcracking seems justified; however not much can be said about the level of homogeneity, i.e. microcracks may still nucleate and propagate in a different rate depending on the location.

The previous comment is related to the second remark, which concerns the extent of the cracks nucleated at the surface. The faces of the surface cracks are highly oxidised and they are therefore easy to evaluate regarding their length by measuring how deep the oxidation has propagated. The crack extent is found to be as long as $0.25 \mathrm{~mm}$ in the $50 \%$ fatigued specimen and $0.4 \mathrm{~mm}$ in the $95 \%$ fatigued specimen, which is about $8 \%$ and $12.5 \%$ of the specimen radius, respectively. These cracks appear longer than the microcracks seen within the bulk; however the verity might be argued since microcracks may be branched or linked together outside the examined cross-section and can therefore be longer than they appear.

The final remark concerns the microcrack linking. As seen in the upper left image of Figure 8, an oxidised surface crack and a microcrack without an oxide layer are found near each other at the surface of the 50\% fatigued specimen. This indicates that these two are not interconnected at this point but probably will be in due time. Therefore, it is speculated that primary crack growth is a consequence of microcrack linking rather than conventional single-crack propagation, which also has been proposed earlier in an investigation done by Haenny and Zambelli. ${ }^{12}$

In summary, it is concluded that CGI should be fairly insensitive to surface effect such as environmental assisted cracking since propagating microcracks nucleate independently in the bulk. On the contrary, the surface effects are not completely discarded because of a possibly higher growth rate than within the bulk. Therefore, it seems plausible that the final macroscopic crack will emanate from a surface crack and rapidly propagate through the pre-cracked bulk, leading to the observed abrupt drop in stress and unloading modulus.

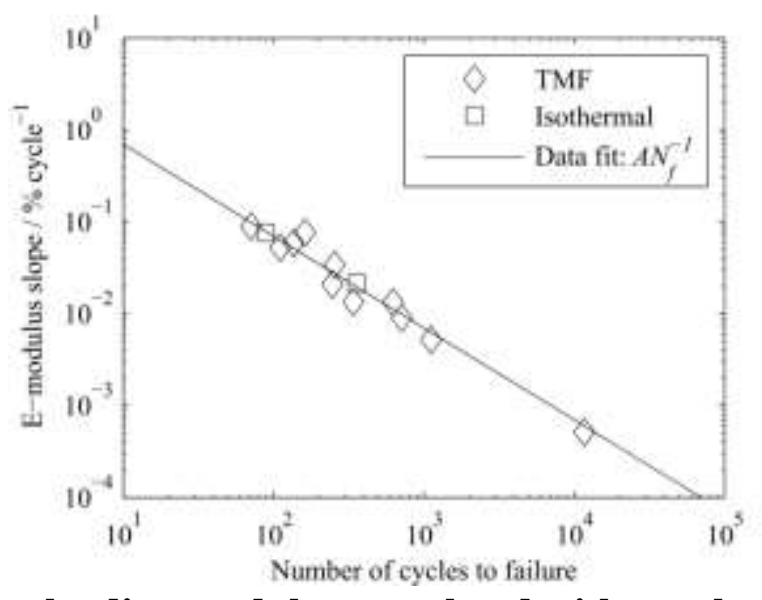

7 Rate of change of unloading modulus correlated with number of cycles to failure $N_{f}$ 


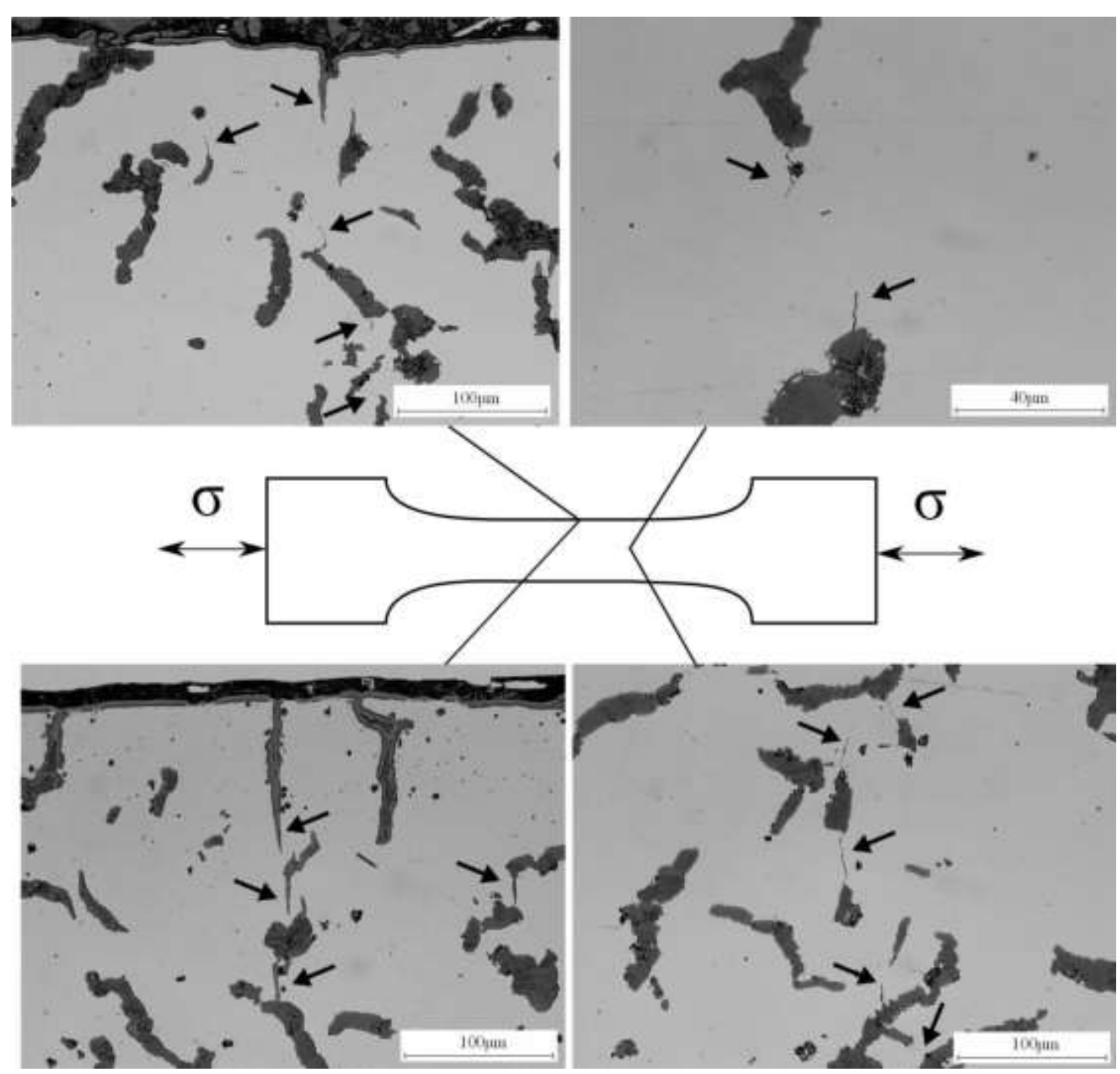

8 Two top images show surface and internal cracks of $50 \%$ fatigued specimen, while two bottom images correspond to $95 \%$ fatigued specimen

\section{Conclusions}

1. Experimental results, both mechanical testing and microstructural investigations, indicate the occurrence of homogenous microcracking in compacted graphite iron during thermomechanical and isothermal low-cycle fatigue. In particular, it is indicated that the evolution of such damage progresses at a constant rate during the major part of the life of the specimen until a critical point where the damage evolution is rapidly accelerated. In conclusion, the proposed life sequence is a two-staged process; (i) constant growth of microcracks independent of each other and (ii) accelerated crack growth due to microcrack linking and the formation of large interlinked cracks.

2. A distinct correlation between the number of cycles to failure $\left(N_{f}\right)$ and the rate of change of the unloading modulus over the numbers of cycles has been identified. This indicates that the damage evolution process in cyclic loading of compacted graphite iron follows a general pattern, i.e. independently of the magnitude of the load variables, while the associated process rate is load variable dependent.

\section{Acknowledgement}

The present study was financiered by Scania CV AB, the Swedish Governmental Agency for Innovation Systems (FFI-2012-03625), and the Swedish Foundation for Strategic Research (SM120014). Agora Materiae and the Strategic Faculty Grant AFM (SFO-MAT-LiU\#2009-00971) at 
Linköping University are also acknowledged. Special thanks are also addressed to the project group at Scania for all their comments and feedback.

\section{References}

1. S. Trampert, T. Gocmez and S. Pischinger: 'Thermomechanical Fatigue Life Prediction of Cylinder Heads in Combustion Engines', J. Eng. Gas Turbines Power, 2008, 130, 012806, 110.

2. J.J. Thomas, L. Verger, A. Bignonnet and E. Charkaluk: 'Thermomechanical design in the automotive industry', Fatigue Fract. Eng. Mater. Struct., 2004, 27, 10, 887-895.

3. S. Ghodrat, A.C. Riemslag, M. Janssen, J. Sietsma and L.A.I. Kestens: 'Measurement and characterization of Thermo-Mechanical Fatigue in Compacted Graphite Iron', Int. J. Fatigue, 2013, 48, 319-329.

4. Z. He, S. Ji and G. Lin: 'Mechanical behaviour of graphite and its adjacent zone to matrix interface in cast iron', Acta Metall. Sin., 1993, 6, 3, 364-368.

5. R.C. Voigt and C.R. jun. Loper: 'Microstructural aspects of fracture in cast irons', Phys. Metall. Cast Iron IV, Proc. 4th Int. Symp., 1989, 293-303.

6. L. Haenny and G. Zambelli: 'Strain mechanisms in grey cast iron', Eng. Fract. Mech., 1983, $18,2,377-387$.

7. G.N.J. Gilbert: 'The components of strain due to deformation of the matrix and due to volume changes in a flake graphite cast iron under uniaxial stress', J. Br. Cast Iron Res. Assoc., 1963, 11, 512-524.

8. J.W. Fash: 'Fatigue crack initiation and growth in gray cast iron', 1980, Fracture Control Program, Report No. 39

9. H. Nisitani and S. Tanaka: 'Initiation and propagation of fatigue crack in cast iron', Trans. Japan Soc. Mech. Eng. Part A, 1985, 51, 465, 1442-1447.

10. H. Pirgazi, S. Ghodrat and L.A.I. Kestens: 'Three-dimensional EBSD characterization of thermo-mechanical fatigue crack morphology in compacted graphite iron', Mater. Charact., 2014, 90, 13-20.

11. P. Hähner, E. Affeldt, T. Beck, H. Klingelhöffer, M. Loveday and C. Rinaldi: 'Validated Code-of-Practice for Thermo-Mechanical Fatigue Testing',2006.

12. L. Haenny and G. Zambelli: 'Crack growth resistance of a gray cast iron', Eng. Fract. Mech., 1985, 21, 3, 529-535. 